\title{
FKBP5 moderation of the relationship between childhood trauma and maladaptive emotion regulation strategies in adolescents
}

\author{
Thorhildur Halldorsdottir ${ }^{a, *}$, Ana Paula Soares de Matos ${ }^{\mathrm{b}, * *}$, Yvonne Awaloff ${ }^{\mathrm{a}}$, \\ Eiríkur Örn Arnarson ${ }^{c}$, W. Edward Craighead ${ }^{\mathrm{d}, 1}$, Elisabeth B. Binder ${ }^{\mathrm{a}, \mathrm{d}, 1}$ \\ a Department of Translational Research in Psychiatry, Max Planck Institute of Psychiatry, Germany \\ ${ }^{\mathrm{b}}$ Faculty of Psychology and Educational Sciences of the University of Coimbra, Portugal \\ c Landspitali-University Hospital, University of Iceland, Faculty of Medicine, School of Health Sciences, Reykjavik, Iceland \\ d Department of Psychiatry and Behavioral Sciences and Department of Psychology, Emory University School of Medicine, USA
}

\section{A R T I C L E I N F O}

\section{Keywords:}

FKBP5

Rumination

Catastrophizing

Childhood trauma

Adolescents

\begin{abstract}
A B S T R A C T
Maladaptive emotion regulation strategies, such as rumination and catastrophizing, are transdiagnostic risk factors for psychopathology. FK506-binding protein 51 (FKBP5) has been found to moderate the relationship between stressful life events and various psychiatric disorders. Given the cross-disorder moderation effect of FKBP5 at the diagnostic level, the aim of the current study was to examine whether the relationship between exposure to childhood trauma and transdiagnostic maladaptive emotion regulation processes would also be moderated by genetic FKBP5 variation in a community sample of adolescents. We hypothesized that adolescent carriers of the FKBP5 CATT haplotype composed of rs9296158, rs3800373, rs1360780, and rs9470080, that has been associated with increased risk for psychiatric disorders in adulthood, would also show higher levels of rumination and catastrophizing. Participants included 1345 genotyped adolescents $\left(\mathrm{M}_{\mathrm{age}}=13.95,64.2 \%\right.$ female; 100\% European Caucasians of Portuguese descent) who completed self-report measures on exposure to childhood trauma and emotion regulation strategies. Genotypes of rs9296158, rs3800373, rs1360780, and rs9470080 were used to estimate the CATT haplotype (carriers versus non-carriers). Consistent with our hypotheses and previous findings, adolescent CATT haplotype carriers with higher levels of childhood trauma endorsed higher levels of both rumination and catastrophizing compared to non-carriers. Given the association of these maladaptive emotion regulation processes and psychiatric disorders, the findings suggest possible psychological mechanisms why FKBP5 haplotype carriers exposed to childhood trauma are more vulnerable to developing a psychiatric disorder later in life.
\end{abstract}

\section{Introduction}

Due to the rising prevalence of depression and anxiety (Mathers and Loncar, 2006), investigating factors associated with increased risk of these disorders in premorbid adolescent populations is important. Adolescence is a high-risk period for the onset of psychiatric disorders due to the accelerated psychological, biological and endocrine development occuring during this time in the lifespan, which interacts with the intensified stress and emotional responsiveness to the social environment (Paus et al., 2008). Accordingly, an increased understanding of risk and resilience factors during this sensitive developmental stage may be crucial in developing effective preventative interventions.
Maladaptive emotion regulation strategies, such as rumination and catastrophizing, have been identified as transdiagnostic risk factors for psychopathology (Gellatly and Beck, 2016; Ottaviani et al., 2016). Rumination refers to the tendency to perseverate on negative cognitions and the potential causes and consequences of distress (NolenHoeksema, 2000; Ottaviani et al., 2016). Catastrophizing, on the other hand, is the tendency to overestimate negative consequences associated with an event (Gellatly and Beck, 2016). Both rumination and catastrophizing have been associated with the onset, maintenance, and recurrence of mood and anxiety disorders (Gellatly and Beck, 2016; Ottaviani et al., 2016).

Overall, risk for psychiatric disorders is associated with

\footnotetext{
* Corresponding author at: Department of Translational Research in Psychiatry, Kraepelinstrasse 2-10, Munich, 80804, Germany.

*** Corresponding author at: Faculty of Psychology and Educational Sciences of the University of Coimbra, Rua do Colégio Novo, Coimbra, 3001-802, Portugal.

E-mail addresses: halldorsdottir@psych.mpg.de (T. Halldorsdottir), apmatos@fpce.uc.pt (A.P.S. de Matos), yvonne_awaloff@psych.mpg.de (Y. Awaloff), eirikur@landspitali.is (E.Ö. Arnarson), ecraigh@emory.edu (W.E. Craighead), binder@psych.mpg.de (E.B. Binder).

${ }^{1}$ These authors contributed equally to the project.
} 
Table 1

Sample demographics.

\begin{tabular}{lllll}
\hline Characteristic & $\begin{array}{l}\text { Homozygous } \\
\text { CATT carrier } \\
\mathrm{N}=114\end{array}$ & $\begin{array}{l}\text { Heterozygous } \\
\text { CATT carrier } \\
\mathrm{N}=530\end{array}$ & $\begin{array}{l}\text { Homozygous } \\
\text { non-carrier } \\
\mathrm{N}=701\end{array}$ & $\begin{array}{l}\text { Total } \\
\mathrm{N}=1345\end{array}$ \\
\hline $\begin{array}{l}\text { Age M(SD) } \\
\text { Sex N(\%) }\end{array}$ & $13.94(.95)$ & $13.91(.88)$ & $13.97(.91)$ & $13.95(.90)$ \\
$\quad$ Females & $76(66.7)$ & $341(64.3)$ & $447(63.8)$ & $864(64.2)$ \\
$\quad$ Males & $38(33.3)$ & $189(35.7)$ & $254(36.2)$ & $481(35.7)$ \\
SES N(\%) & & & & \\
$\quad$ Low & $66(57.9)$ & $303(57.2)$ & $402(57.3)$ & $771(57.3)$ \\
$\quad$ Medium & $26(22.8)$ & $144(27.2)$ & $178(25.4)$ & $348(25.9)$ \\
$\quad$ High & $22(19.3)$ & $79(14.9)$ & $109(15.5)$ & $210(15.6)$ \\
Childhood trauma M & $31.75(7.64)$ & $32.55(8.74)$ & $32.25(8.33)$ & $32.33(8.44)$ \\
$\quad(S D)$ & & & & \\
$\quad$ Rumination M(SD) & $11.48(3.83)$ & $11.31(3.63)$ & $11.54(3.74)$ & $11.44(3.70$ \\
Catastrophizing M & $9.20(3.71)$ & $8.87(3.66)$ & $9.01(3.76)$ & $8.97(3.72)$ \\
$\quad(S D)$ & & & &
\end{tabular}

Note: Socioeconomic status (SES) based on the parents' profession and education level according to Simões classification (Simões, 1995). SES = socioeconomic status.

a Seventeen participants were missing SES information (4 heterozygous CATT carriers and 13 homozygous non-carriers).

environmental and genetic factors, as well as their interactions (Halldorsdottir and Binder, 2017). One of the most consistent risk factors for psychopathology in adulthood is exposure to childhood trauma, and various genetic polymorphisms have been described to moderate this relationship. Among these genetic markers are variants within the gene encoding the FK506-binding protein 51 FKBP5, which plays an important role in the hypothalamic-pituitary-adrenal (HPA) axis through its regulatory role of glucocorticoid receptor sensitivity (Binder, 2009). Gene-by-environment interaction $(\mathrm{G} \times \mathrm{E})$ studies have found that minor allele carriers of a haplotype derived of 4 variants (rs9296158, rs3800373, rs1360780, and rs9470080), referred to as CATT carriers, are at greater risk of developing a range of psychiatric disorders in adulthood following childhood trauma relative to major allele carriers (Zannas et al., 2016). Only few studies have, however, examined the moderating role of FKBP5 on transdiagnostic psychological risk factors for psychopathology in youth, especially adolescents (Comasco et al., 2015; Isaksson et al., 2016). Toward bridging this gap, the current study investigated the combined predictive effect of this FKBP5 haplotype and exposure to childhood trauma on the maladaptive emotion regulation strategies described above. Given that this FKBP5 haplotype has shown moderating effects of childhood adversities across psychiatric disorders, we hypothesized that these transdiagnostic emotion regulation processes would also be affected in that CATT haplotype carriers exposed to higher levels of childhood trauma would present with the higher level of rumination and catastrophizing compared to non-carriers.

\section{Method}

\subsection{Participants}

Participants were drawn from a larger study comprising 1459 students between the ages $12-17$ and their parents recruited from 31 schools in Portugal in 2011-2012 and 2013-2014. A total of 1345 genotyped adolescents between the ages 12-17 $\left(\mathrm{M}_{\mathrm{age}}=13.95,64.2 \%\right.$ female; $100 \%$ European Caucasians of Portuguese nationality) completed the measures required for the present study (see Table 1 for sample characteristics). All subjects' parents completed consent forms and students completed assent forms prior to participation. Trained research assistants (master's students and licensed psychologists) administered the assessment battery and extracted saliva in the school classroom setting. Genetic samples were genotyped at the Max Planck Institute of Psychiatry (MPIP) in Germany. De-identified phenotypic data $^{1}$ were also supplied to the researchers at MPIP for analyses. All protocols were approved by the Portuguese National Committee for Data Protection and the Ethical Committee of the Faculty of Psychology and Educational Psychology of the University of Coimbra.

\subsection{Psychometric measures}

The Childhood Trauma Questionnaire (CTQ; Bernstein et al., 2003) was used to assess childhood trauma. Participants rated each item about his/her childhood experience on a 5-point scale (Never true to Always true). In the current study, a total score of the abuse (emotional, physical, and sexual abuse) and neglect (emotional and physical neglect) subscales was used (Cronbach's $\alpha=0.745$ ), with higher ratings indicating higher levels of childhood trauma. The psychometric properties of the CTQ were adequate in Portuguese samples (Dias et al., 2014).

The Cognitive Emotion Regulation Questionnaire (CERQ; Garnefski et al., 2001) assessed self-reported rumination (4 items on 1-5 scale, Never to Always; Cronbach's $\alpha=0.792$ ), and catastrophizing (4 items on 1-5 scale, Never to Always; Cronbach's $\alpha=0.794$ ). Higher scores indicate greater frequency of engagement in the maladaptive cognitive strategy. The CERQ has been translated to Portuguese and its psychometric properties have proven adequate (Castro et al., 2013).

\subsection{Genotyping methods for the FKBP5 gene}

DNA was extracted from saliva from each participant using Oragene Saliva kits (DNA Genotek). Genotyping was performed with the Infinium Global Screening Array protocol (for a detailed description of the protocol, see www.illumina.com). Single-nucleotide polymorphisms (SNPs) with Hardy-Weinberg equilibrium (HWE) $<0.0001$, minor allele frequency $(\mathrm{MAF})<0.05$ and call rate $<95 \%$ were excluded for quality control. With the SNPs surviving this quality control, imputation for additional variants was performed using IMPUTE version 2 (Howie, Donnelly, \& Marchini, 2009) and the 1000 Genomes Project reference genome. Imputed SNPs were excluded if their posterior probability averages were less than $90 \%$ for the most likely imputed genotype (INFO $\geq 0.9$ ). After the imputation process, SNPs with call rate $<98 \%$, HWE $p<0.0001$ and MAF $<0.05$ were excluded. This yielded a total of 3.779.545 SNPs.

The genotypes of four SNPs of the FKBP locus (rs9296158, rs3800373, rs1360780, and rs9470080) in linkage disequilibrium $\left(r^{2}>0.78\right.$ for all SNPs) previously found to be associated with a host of psychiatric disorders (Zannas et al., 2016) were use to compute the number of CATT haplotype (carriers versus non-carriers) copies using PLINK (version 1.07; Purcell et al., 2007), an open-source whole genome association analysis software program. Consistent with the previous literature, the CATT haplotype was coded based on the dominant model $(0=$ homozygous non-carrier; $1=$ homozygous or heterozygous CATT haplotype carrier). See Table 1 for genotype frequencies. Principal components (PCAs) to account for population stratification were calculated using genome-wide complex trait analysis (Yang et al., 2013). Three clusters were identified and, thus, the first 3 PCAs were included in all analyses to control for population stratification.

\subsection{Data analysis}

Chi-square and $t$-test analyses were used to examine genotype differences and regression analyses were conducted to investigate the association of demographic and clinical characteristics with the

\footnotetext{
${ }^{1}$ Each participant was assigned a subject number, and the psychometric measures were scored and put into databases with only subject numbers as identifiers. Original measures with individual identifying data were kept in double locked storage in the research offices of the principal investigator (APM) in Portugal.
} 
dependent variables (i.e., rumination and catastrophizing). The main effects and interaction of exposure to childhood trauma and CATT haplotype on rumination and catastrophizing were examined with multivariate analysis of variance (MANOVA) using SPSS software for Windows (IBM SPSS version 22). The interaction was composed of the cross-product term for exposure to childhood trauma (CTQ total score $) \times$ CATT haplotype $(0=$ non-carrier; $1=$ heterozygous or homozygous CATT haplotype carrier). As secondary analyses, we also examined a three-way interaction including biological sex (i.e., childhood trauma $\times$ CATT haplotype $\times$ biological sex). In accordance with the recommendation of Keller (2014), significant group differences and characteristics significantly associated with the dependent variables and their interaction with the independent variables (i.e., CTQ total score and CATT haplotype) were also included as covariates in all analyses (Keller, 2014). To correct for multiple testing, two-tailed $p<0.025$ was considered significant. For significant interactions, a test of region of significance (RoS) was performed using an SPSS macro developed by Prof. Johannes Ullrich. Power analyses indicated the ability to detect a small effect $\left(f^{2} \leq 0.10\right)$ with an alpha probability of 0.025 and power of $80 \%$.

\section{Results}

Demographic and clinical characteristics of the sample are presented in Table 1. No significant differences were noted based on FKBP5 genotype. Higher levels of rumination and catastrophizing were however observed among participants with lower socioeconomic status (SES) status $(p<0.001)$. Additionally, females endorsed higher levels of rumination and catastrophizing than males $(p<0.001)$. SES and biological sex and their interaction with the independent variables (i.e., exposure to childhood trauma and CATT haplotype) were therefore controlled for in all analyses (Keller, 2014). Consistent with previous findings (Garnefski et al., 2001), rumination and catastrophizing were significantly correlated ( $r=0.587, p<0.001)$.

Controlling for sex and SES, gene-environment correlation revealed a nonsignificant correlation between CATT haplotype and exposure to childhood trauma ( $r \mathrm{GE}=0.014, p=0.615$ ). Partial correlations, adjusting for sex and SES, revealed a significant correlation between exposure to childhood trauma and rumination for both CATT haplotype carriers and non-carriers. The correlation however was higher among CATT haplotype carriers $(r=0.231, p<0.001)$ than non-carriers $(r=0.168, p<0.001)$. The same pattern was observed for the correlation between exposure to childhood trauma and catastrophizing (CATT haplotype carriers: $r=0.324, p<0.001$; non-carriers: $r=0.294, p<.001$ ). The significant correlations were to be expected due to the positive relationship between exposure to childhood trauma and maladaptive emotion regulation strategies, in addition to the large sample size in the current study.

\subsection{Interaction between FKBP5 genotype and childhood abuse predicting rumination}

Exposure to childhood trauma $(\mathrm{F}(1)=42.706, \quad p<0.001$, $\eta^{2}=0.031$ ) significantly predicted rumination but CATT haplotype did not $\left(\mathrm{F}(1)=1.454, p=0.228, \eta^{2}=0.001\right)$. Specifically, youth exposed to higher levels of childhood trauma endorsed higher levels of rumination than did youth with lower levels of exposure to childhood trauma. The interaction between exposure to childhood trauma and CATT haplotype also significantly predicted rumination $(\mathrm{F}(1)=6.418$, $\left.p=0.011, \eta^{2}=0.005\right)$. Specifically, CATT haplotype carriers exposed to higher levels of childhood trauma showed higher levels of rumination than haplotype carriers exposed to lower levels of childhood trauma and non-carriers with and without a history of childhood trauma (see Fig. 1A). Interestingly, higher exposure to childhood trauma was associated with lower rumination ratings in non-carriers. The interaction between exposure to childhood trauma and CATT haplotype predicting rumination was not further moderated by biological sex $(p=0.978)$.

For the two-way interaction, RoS analyses revealed that the interaction of FKBP5 haplotype and exposure to childhood trauma predicting rumination was significant across the entire range of the CTQ score. Specifically, the regression analyses extrapolated that the interaction would be significant if the exposure to childhood trauma was above 4.205 .

\subsection{Interaction between FKBP5 genotype and childhood abuse predicting catastrophizing}

The main effect for exposure to childhood trauma $(\mathrm{F}(1)=137.296$, $\left.p<0.001, \eta^{2}=0.093\right)$ was significantly associated with catastrophizing. That is, higher levels of catastrophizing were endorsed by adolescents exposed to higher levels of childhood trauma. The main effect of CATT haplotype ( $\mathrm{F}(1)=0.290, p=0.590, \eta^{2}<0.001$ ) on catastrophizing was not significant. The interaction between exposure to childhood trauma and CATT haplotype $(\mathrm{F}(1)=5.949, p=0.015$, $\eta^{2}=0.005$ ) significantly predicted catastrophizing in that higher levels of catastrophizing were endorsed by CATT haplotype carriers exposed to higher levels of childhood trauma compared to haplotype carriers with lower levels of exposure to childhood trauma and non-carriers independent of the exposure to childhood trauma (see Fig. 1B). The three-way interaction with childhood trauma, CATT haplotype, and biological sex $(p=0.107)$ did not significantly predict catastrophizing.

Again, RoS analyses for the two-way interaction showed that the interaction between FKBP5 haplotype and exposure to childhood trauma predicting catastrophizing was significant across the entire range of the CTQ, namely the interaction was significant if the exposure to childhood trauma was 4.065 or above.

\section{Discussion}

We found that FKBP5 haplotype interacted with exposure to childhood trauma to predict both rumination and catastrophizing in adolescents. Consistent with our hypotheses and previous findings (Zannas et al., 2016), CATT haplotype carriers exposed to higher levels of childhood trauma reported higher levels of both rumination and catastrophizing, whereas this pattern was not observed in non-carriers. As noted previously, rumination and catastrophizing have been identified as transdiagnostic risk factors for psychopathology (Garnefski et al., 2001; Gellatly and Beck, 2016; Ottaviani et al., 2016). Accordingly, the findings may suggest that adolescent CATT haplotype carriers exposed to childhood trauma display altered emotion regulation, and they are therefore more susceptible to developing a psychiatric disorder than non-carriers independent of exposure to childhood trauma. Longitudinal studies are needed, however, to determine whether this is indeed the sequence of events. Such findings could have important clinical implications in terms of identifying at-risk individuals for psychopathology in premorbid populations.

As noted above, adolescence is a crucial and fragile developmental period in multiple ways, including brain development (Paus et al., 2008). The combination of risk-increasing genes and environmental influences likely results in pathophysiological disturbance in molecular pathways and consequently may influence the neural circuits associated with psychopathology. Pertinent to the current findings, brain regions associated with emotion processing and memory, such as the amygdala and hippocampus, are believed to be involved in FKBP5-related vulnerability to psychopathology (Zannas et al., 2016). Interestingly, rumination has also been associated with increased activation of the default mode network, which includes the amygdala and hippocampus (Belzung et al., 2015). Future studies are needed to determine whether this association between these brain regions and rumination and catastrophizing are moderated by FKBP5 genotype. Such an increased understanding of the underlying pathophysiology could lead to more 

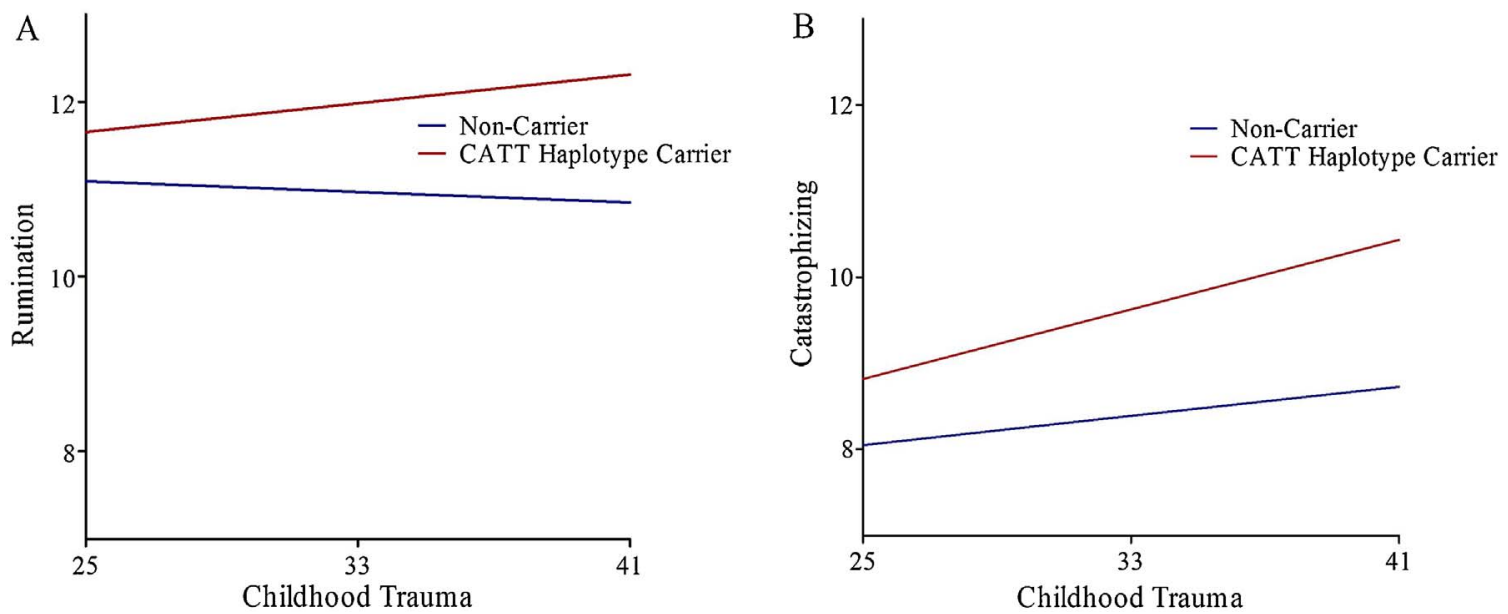

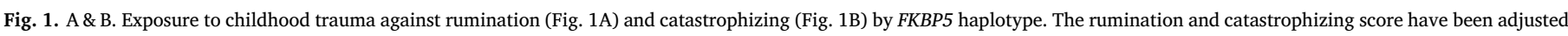
for socioeconomic status and biological sex.

targeted prevention efforts and treatments.

Strengths of the study include the relatively large sample size and the incorporation of transdiagnostic processes associated with the development of psychiatric disorders that were dimensionally conceptualized as recommended in the National Institute of Mental Health's Research Domain Criteria and DSM-5. The current study, however, also has several limitations. For instance, all of the participants were Caucasian; as such, generalizability to other ethnicities is limited. Furthermore, our analyses were limited to rumination and catastrophizing, which have been associated primarily with internalizing disorders. Future studies should investigate the relationship between genetic and environmental factors with other transdiagnostic processes associated with the development of externalizing disorders and psychotic symptoms. Lastly, the study investigated a composite score of the exposure to emotional, physical and sexual abuse and emotional and physical neglect. It is possible that specific subtypes of childhood trauma interact differently with the FKBP5 genotype in predicting maladaptive emotion regulation strategies. Further delineation of potential moderation effects of FKBP5 based on the type of childhood trauma shaping the risk for psychopathology will therefore be needed.

To conclude, the current study brings novelty and expands upon previous $\mathrm{G} \times \mathrm{E}$ studies by examining the moderating effect of FKBP5 and stressful life events on transdiagnostic risk factors of psychopathology, namely rumination and catastrophizing. The findings may have important clinical implications towards a neurobiological informed nosology and more targeted identification of at-risk adolescents given that such maladaptive emotion regulation strategies are transdiagnostic precursors of psychiatric disorders.

\section{Role of funding source}

This work was supported by the Fundação para a Ciência e a Tecnologia (Portuguese Foundation for Science and Technology) within the project "Prevention of depression in Portuguese adolescents: efficacy study of an intervention with adolescents and parents" (PTDC/ MHC-PCL/4824/2012) and in part by an ERC starting grant, $\mathrm{G} \times \mathrm{E}$ molmech, grant number 281338 to EBB and the Fuqua family foundations.

\section{Conflict of interest/disclosure}

WEC is a board member of Hugarheill ehf, an Icelandic company dedicated to the prevention of depression, and he receives book royalties from John Wiley \& Sons. His research is also supported by the NIH, the Mary and John Brock Foundation, and the Fuqua family foundations. He is a consultant to the George West Mental Health Foundation that oversees Skyland Trail, a residential treatment facility in Atlanta, Georgia.

EBB is co-inventor on FKBP5: a novel target for antidepressant therapy, European Patent\# EP 1687443 B1, and receives a research grant from Böhringer-Ingelheim for a collaboration on functional investigations of FKBP5.

TH, APM, YA, and EOA have nothing to disclose.

\section{Acknowledgements}

We would like to thank the adolescents who gave their time to participate in this study. We would also like to thank the assessors at the University of Coimbra and technicians involved in the processing of the genetic data at the Max Planck Institute of Psychiatry.

\section{References}

Belzung, C., Willner, P., Philippot, P., 2015. Depression: from psychopathology to pathophysiology. Curr. Opin. Neurobiol. 30, 24-30. http://dx.doi.org/10.1016/j.conb. 2014.08.013.

Bernstein, D.P., Stein, J.A., Newcomb, M.D., Walker, E., Pogge, D., Ahluvalia, T., Desmond, D., 2003. Development and validation of a brief screening version of the Childhood Trauma Questionnaire. Child Abuse Neglect 27 (2), 169-190.

Binder, E.B., 2009. The role of FKBP5, a co-chaperone of the glucocorticoid receptor in the pathogenesis and therapy of affective and anxiety disorders. Psychoneuroendocrinology 34 (Suppl. 1), S186-S195. http://dx.doi.org/10.1016/j. psyneuen.2009.05.021.

Castro, J., Soares, M.J., Pereira, A.T., Chaves, B., Macedo, A., 2013. Questionário da Regulação Emocional Cognitiva: Validação para a População Portuguesa Cognitive Emotional Regulation Questionnaire: Validation of the Portuguese Version. Psiquiatria Clinica 34, 101-109.

Comasco, E., Gustafsson, P.A., Sydsjo, G., Agnafors, S., Aho, N., Svedin, C.G., 2015. Psychiatric symptoms in adolescents: FKBP5 genotype-early life adversity interaction effects. Eur. Child Adolesc. Psychiatry 24 (12), 1473-1483. http://dx.doi.org/10. 1007/s00787-015-0768-3.

Dias, A., Sales, L., Carvalho, A., Castro-Vale, I., Kleber, R., Cardoso, R.M., 2014. Estudo de propriedades psicométricas do Questionário de Trauma de Infância?Versão breve numa amostra portuguesa não clínica. Laboratório de Psicologia 11, 103-120.

Garnefski, N., Kraaij, V., Spinhoven, P., 2001. Negative life events, cognitive emotion regulation and emotional problems. Person. Individ. Diff. 30 (8), 1311-1327.

Gellatly, R., Beck, A.T., 2016. Catastrophic thinking: a transdiagnostic process across psychiatric disorders. Cogn. Ther. Res. 1-12.

Halldorsdottir, T., Binder, E.B., 2017. Gene x environment interactions: from molecular mechanisms to behavior. Annu. Rev. Psychol. 68, 215-241. http://dx.doi.org/10. 1146/annurev-psych-010416-044053.

Howie, B.N., Donnelly, P., Marchini, J., 2009. A flexible and accurate genotype imputation method for the next generation of genome-wide association studies. PLoS Genet. 5 (6), e1000529. http://dx.doi.org/10.1371/journal.pgen.1000529.

Isaksson, J., Comasco, E., Aslund, C., Rehn, M., Tuvblad, C., Andershed, H., Nilsson, K.W., 2016. Associations between the FKBP5 haplotype, exposure to violence and anxiety in females. Psychoneuroendocrinology 72, 196-204. http://dx.doi.org/10.1016/j. psyneuen.2016.07.206.

Keller, M.C., 2014. Gene $\times$ environment interaction studies have not properly controlled 
for potential confounders: the problem and the (simple) solution. Biol. Psychiatry 75 (1), 18-24.

Mathers, C.D., Loncar, D., 2006. Projections of global mortality and burden of disease from 2002 to 2030. PLoS Med. 3 (11), e442.

Nolen-Hoeksema, S., 2000. The role of rumination in depressive disorders and mixed anxiety/depressive symptoms. J. Abnorm. Psychol. 109 (3), 504.

Ottaviani, C., Thayer, J.F., Verkuil, B., Lonigro, A., Medea, B., Couyoumdjian, A., Brosschot, J.F., 2016. Physiological concomitants of perseverative cognition: a systematic review and meta-analysis. Psychol. Bull. 142 (3), 231-259. http://dx.doi. org/10.1037/bul0000036.

Paus, T., Keshavan, M., Giedd, J.N., 2008. Why do many psychiatric disorders emerge during adolescence? Nat. Rev. Neurosci. 9 (12), 947-957. http://dx.doi.org/10. 1038/nrn2513.

Purcell, S., Neale, B., Todd-Brown, K., Thomas, L., Ferreira, M.A.R., Bender, D., Daly, M.J., 2007. PLINK: a tool set for whole-genome association and population-based linkage analyses. Am. J. Hum. Genet. 81 (3), 559-575.

Yang, J., Lee, S.H., Goddard, M.E., Visscher, P.M., 2013. Genome-wide complex trait analysis (GCTA): methods, data analyses, and interpretations. Methods Mol. Biol. 1019, 215-236. http://dx.doi.org/10.1007/978-1-62703-447-0_9.

Zannas, A.S., Wiechmann, T., Gassen, N.C., Binder, E.B., 2016. Gene-stress-epigenetic regulation of FKBP5: clinical and translational implications.

Neuropsychopharmacology 41 (1), 261-274. http://dx.doi.org/10.1038/npp.2015 235. 